\title{
Indoleamine 2,3-dioxygenase as a novel therapeutic target for Huntington's disease
}

\author{
Fanni A. Boros ${ }^{\mathrm{a}}$, Péter Klivényi ${ }^{\mathrm{a}}$, József Toldi ${ }^{\mathrm{b}, \mathrm{c}}$ and László Vécsej ${ }^{\mathrm{a}, \mathrm{c}}$
}

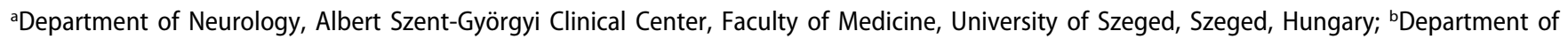
5 Physiology, Anatomy and Neuroscience, Faculty of Science and Informatics, University of Szeged, Szeged, Hungary; ' $M T A-S Z T E$ Neuroscience

\section{ABSTRACT}

Introduction: Huntington's disease (HD) is an autosomal dominant, neurodegenerative disorder. Despite the severe motor, psychiatric and cognitive symptoms and the great socioeconomic burden caused by the disease, available treatment is mainly symptomatic.

The kynurenine pathway (KP) is the main metabolic route of tryptophan degradation, in the course of which several neuroactive compounds are generated. The imbalance of the neurotoxic and neuroprotectant metabolites can lead to excitotoxicity and overproduction of reactive oxygen species, which both contribute to the progression of HD. Indoleamine 2,3-dioxygenase 1 (IDO1) is a key enzyme of the $\mathrm{KP}$ that has various immune modulatory roles.

Areas covered: Current knowledge of the involvement of KP in HD pathogenesis with a particular focus on IDO1. By reviewing the diverse roles of the enzyme in kynurenine production, immune modulation, and serotonin metabolism, we elucidate the factors that make this enzyme a therapeutic target.

Expert opinion: Due to the complexity of HD and the various effects that IDO1 exerts, targeting this enzyme, while highly profitable, may be a great challenge. Through IDO1 activity, neurodegeneration, inflammatory processes and depressive symptoms, often related to HD, can be modulated. Ongoing trials of IDO1 inhibitors in other areas of medicine offer advantages for initiating approaches toward this enzyme as a therapeutic target.
ARTICLE HISTORY

Received 13 July 2018

Accepted 13 November

2018

\section{KEYWORDS}

Huntington's disease; kynurenine pathway; indoleamine 2;

3-dioxygenase; IDO1; excitotoxicity; inflammation; depression

\section{Introduction} symptomatic.
Huntington's disease (HD) is a hereditary, autosomal dominant, progressive neurodegenerative disease with various motor, psychiatric and cognitive symptoms (for a review see [1]), that affects 6-13 individuals out of 100,000 [2]. Despite the severity of the disease, available therapy for HD is mainly

The kynurenine pathway (KP) is the main route of tryptophan (Trp) metabolism with several metabolites that have been identified to be neuroactive. As imbalances of neuroprotectant and neurotoxic metabolites have been proposed to be key factors in $\mathrm{HD}$, elements of the KP might offer potential therapeutic targets for the disease.

In this review, we will consider indoleamine 2,3-dioxygenase (IDO), the first and rate-limiting enzyme of the $\mathrm{KP}$, as a novel therapeutic target for HD treatment. The modulation of IDO activity provides opportunity for restoring the balance between neuroprotectant and neurotoxic metabolites of the $\mathrm{KP}$ and, due to the diverse functions of IDO in immune regulation, it also offers possible ways to interfere with altered inflammatory processes in HD.

Here we summarize the available data on KP alterations that are related to $\mathrm{HD}$, with particular attention to IDO. We would like to emphasize that due to the diverse effects of IDO functions and the complexity of HD pathogenesis, there are several junctions between $\mathrm{HD}$ and $\mathrm{KP}$, that make IDO a potential therapeutic target for HD treatment. In order to explore these possibilities, we will outline the pathological mechanisms underlying HD and summarize available data on $\mathrm{KP}$ alterations in the disease.

\subsection{A brief overview of Huntington's disease pathogenesis}

The genetic background of HD was elucidated in 1993 with the identification of an expansion of CAG trinucleotide repeats in exon 1 of the Huntingtin (HTT) gene [3]. The trinucleotide repeat expansion leads to the formation of an elongated polyglutamine sequence (polyQ) at the $\mathrm{N}$ terminal region of Huntingtin protein (HTT) [3]. Although HTT has been shown to interact with a large number of various proteins, among them components of the cytoskeleton and plasma membrane, synaptic vesicles and transcriptional factors [4], its detailed physiological function is still unknown. Due to the presence of an elongated polyQ sequence (over $35 \mathrm{Glu}$ ), the mutant protein becomes insoluble and forms aggregates, both in the cytoplasm and in the nucleus [5]. It seems that the HTT protein with extended polyQ is toxic to neurons [6], and an increasing body of evidence suggest that the formation of inclusion bodies is a coping mechanism of the cells against the mutant 
Article highlights

- A growing body of evidence supports the involvement of the KP in HD pathogenesis.

- The upregulated state of the pathway results in the excessive production of neurotoxic metabolites such as QUIN and 3-HK and a decrease in the level of the neuroprotective KYNA.

- ID01 catalyzes the first step of the KP and has various roles in modulating immune responses.

- Inhibition of the first step of the KP was proven to be neuroprotective, as it shifted the pathway toward the formation of KYNA and reduced QUIN-induced toxicity by decreasing the levels of 3-HK.

- Adjusting IDO1 activity could be beneficial in HD therapy as it would restore the balance between neuroprotective and neurotoxic kynurenine compounds, modulate the upregulated inflammatory state and alleviate depressive symptoms often present with the disease.

- Testing IDO1 inhibitor compounds that have been proven to be safe in other areas of medicine could be a cost and time effective way of trying novel therapeutic agents for HD.

This box summarizes key points contained in the article.

HTT protein and not an early sign of cell death [7]. Cells with inclusion bodies survive longer than those [8]. Neurodegeneration in HD is most prominent in the striatum, mainly due to the selective loss of the GABAerg medium spiny neurons (MSNs) [9]. These neurons receive glutamatergic and dopaminergic inputs from the cortex and substantia nigra, respectively, and also from striatal interneurons [10], and project to several components of the basal ganglia (for a review

80 see [11]). MSNs express various receptors of which the $\mathrm{N}$-methyl-D-aspartate (NMDA) glutamate receptor seems to have a cardinal role in the development of HD [12].

Several cellular pathomechanisms have been identified to contribute to the development and progression of the disease (for a review see [13]). These involve excitotoxicity, mitochondrial dysfunction, and associated impairment in energy metabolism, excessive production of reactive oxygen species (ROS), lipid, and protein oxidation. Whether these pathological mechanisms are causes or effects of each other cannot be

90 clearly distinguished as these processes are tightly connected and their malfunctions lead to a vicious circle.

The occurrence of excitotoxicity (which refers to neuronal death due to the activation of amino acid receptors [14]) in HD pathogenesis was initially proposed based on observations by Coyle and Schwarcz that the injection of kainic acid (KA) into the striatum of rats resulted in neuropathological and neurochemical features similar to those seen in HD. One of the most prominent features which develop upon KA administration was the selective loss of the striatal MSNs, which is also seen in HD [15]. However, this KA-induced model of HD did not spare interneurons of the striatum, which are known to be preserved in HD neurodegeneration. In an attempt to establish a more accurate HD model Beal and colleagues used intrastriatal quinolinic acid (QUIN) administration [16]. QUIN, 105 a known NMDA receptor agonist [17], is generated from Trp via the KP. Injection of QUIN into rat striatum resulted in the selective degeneration of striatal MSNs accompanied by the survival of interneurons [16].

The finding that striatal degeneration is mainly due to the receptors [9], and that the decrease of NMDA receptors in the striatum can be identified even in early, presymptomatic stages of the disease $[18,19]$ supported the notion that neurons that express high level of NMDA receptors show increased vulnerability to degeneration [19]. It has been proposed that the increased neuronal sensitivity might be a consequence of mutant HTT-increased expression of the NR1/NR2B subunit combinations of the NMDA receptors found on the striatal MSNs [20]. The role of excitotoxicity in HD pathogenesis is further strengthened by reports of beneficial effects of the GABAergic and antiexcitotoxic valproate on the survival and locomotory activity in a transgenic mouse model of the disease [21].

Besides excitotoxicity, disturbance in energy metabolism also contributes to the degenerative changes in HD. Early findings of Stahl and colleagues revealed a significant decrease in the activity of the succinate dehydrogenase $(\mathrm{SDH}$, an enzyme directly linked to complex II of the electron transport chain (ETC)) in postmortem HD brain samples [22]. In accordance with these findings, injection of the SDH inhibitor, malonate, into rat striatum caused striatal lesions, which, however, could be prevented by the systematic implementation of the NMDA receptor antagonist MK-801 [23]. These results suggest a link between energy metabolism disturbance and excitotoxicity in HD.

Further evidence for energy disturbances in HD was reported by Jenkins and colleagues, who found elevated lactate levels - an in vivo biomarker of impaired energy metabolism in the occipital cortex of HD patients. Moreover, the degree of elevation of lactate levels was found to be correlated with disease duration [24]. A few years later, the same research group found that oral administration of an essential cofactor of the ETC, coenzyme QI0 (CoQ10) resulted in a decrease in cerebral lactate levels in HD patients [25]. These findings supported the hypothesis that associates energy disturbances with $\mathrm{HD}$ and gave hope of developing HD treatments by exploring this aspect. Indeed, CoQ10 in combination with the NMDA receptor antagonist remacemide has been proven to remedy motor symptoms and abnormal weight-gain in the transgenic mouse HD model [26]. This combination treatment also improved survival and decreased brain atrophy and striatal aggregate number with a consistently lower rate of neuron loss [27]. The positive results in animal models supported testing CoQ10 and remacemide treatment in human HD patients, and a multicenter, randomized, placebo-control study involving 374 HD patients was conducted [28]. Although a trend toward slower disease progression was observed, significant differences between the group of patients treated with CoQ10 and the placebo group was not achieved [28].

Dysfunctional mitochondria can also cause imbalances in ROS production and removal, leading to excessive protein, lipid, and DNA oxidation which damages cells. In HD, mutant HTT protein exacerbates ROS production [29]. Besides ROS, reactive nitrogen species (RNSs) are also culprits in the oxidative damage observed in $\mathrm{HD}$ [30]. The $\mathrm{Ca}^{++}$increase caused by NMDA receptor activation induces neural nitric oxide synthase (nNOS) leading to nitric oxide (NO) formation, which can contribute to the generation of RNSs [5]. Through the inhibition of ETC complexes II and III, NO itself contributes to free radical production that can lead to severe ATP depletion [30]. 
Besides excitotoxicity, mitochondrial disturbances and oxidative stress, inflammatory processes are also believed to be related to neuronal damage in HD. Accumulation of activated microglia has been reported in various regions of the HD brain [31], and the overactivation and dysregulation of these cells were found to correlate with the severity of the disease [32]. Reactive microglia in HD expressing complement proteins and inflammation markers in high levels, likely contribute to neurodegeneration [33]. Earlier findings in Alzheimer's disease $(A D)$, that $\beta$-amyloid triggered complement activation, suggest

180 that a similar scenario might take place in HD, such as the mutant HTT protein causing aggravated activation of the complement system [33]. There are supporting data available that inflammation might affect not only the central nervous system (CNS) but also the periphery: elevated $C$ reactive protein (CRP), neopterin, malondialdehyde and 4-hydroxynoenal levels in blood samples of HD patients indicate a persistent inflammatory state and high level of oxidative stress, possibly reflecting neural damage and removal of the damaged cells in the CNS [20]. Furthermore, increases were found in the levels of IL23 and the soluble human leucocyte antigen-G in parallel with increasing disease severity [34]. Therefore, the assessment of the use of anti-inflammatory drugs in HD therapy is clearly worth consideration.

Among the psychiatric symptoms of the disease, depression is detected strikingly frequently, affecting 40-50 percent of the patients [35]. Presumably at least partly related to this, suicide among HD patients is over five times more common than in the general population [35], causing great concern among patients, family, and caretakers.

\section{The kynurenine pathway}

\subsection{A general overview of the kynurenine pathway}

The KP is the main route of Trp metabolism outside of protein synthesis, both in the periphery and in the CNS [36] (Figure 1). The first and rate-limiting step in the pathway is the conversion of Trp to $\mathrm{N}$-formyl-L-kynurenine either by tryptophan 2,3-dioxygenase (TDO) or by one of two IDO enzymes (IDO1 and IDO2). TDO is mainly expressed in the liver regulating systemic Trp levels. IDO is expressed in several cell types of the immune system (monocytes, macrophages (MAs), dendritic cells (DCs) and microglia) and its expression can be induced by various inflammatory signals, such as interleukins (ILs), tumor necrosis factors (TNFs) and interferons (IFNs), of which its main inducer is IFN $\gamma$ [37].

Trp is transported into the CNS through the blood-brainbarrier (BBB) by the large neutral amino acid transporter [38], and once in the CNS, it can be converted into serotonin and melatonin. However, in the CNS the vast majority of Trp more than 95\% [39] - enters the KP.

The product of the first enzymatic conversion of the KP, $\mathrm{N}$-formyl-L-kynurenine is converted by formamidase into L-kynurenine (L-KYN). L-KYN represents an important branch point of the pathway as it can be metabolized alternatively into kynurenic acid (KYNA), or anthranilic acid (AA), or 3-hydroxykynurenine (3-HK). KYNA is synthesized from L-KYN by kynurenine aminotransferases (KATs). There are four subtypes of KATs (KATI-IV) [40] that can catalyze the conversion. The principal enzyme from these in the human CNS is KATII $[40,41]$. Besides KATs, L-KYN can also be metabolized by the kynureninase enzyme (KYNU) and kynurenine 3-monooxygenase (KMO) forming $\mathrm{AA}$ or $3-\mathrm{HK}$, respectively. $3-\mathrm{HK}$ can be metabolized into xanthurenic acid (XA) by KATs, and both AA and 3-HK can be converted into 3-hydroxyanthranilic acid (3-HAA), a metabolite which is a free radical generator (like other compounds of the pathway, see below) [20]. 3-HAA transforms into the unstable 2-amino-3-carboxymuconate-semialdehyde (ACMS) by 3-hydroxyanthranilate oxidase (3-HAO). ACMS can give rise to either picolinic acid (PIC) by conversion via aminocarboxymuconatesemialdehyde decarboxylase (ACMSD) or to QUIN, a NAD ${ }^{+}$and $\mathrm{NADP}^{+}$precursor metabolite, via a non-enzymatic conversion.

In summary, several steps of the KP produce metabolites that directly or indirectly affect neuron survival. KYNA is a known antagonist of NMDA receptors [42] therefore it is a promising therapeutic compound against glutamateinduced excitotoxicity. In addition to NMDA receptors, KYNA has also been reported to be antagonist of a7nicotinic acetylcholine receptors ( $\mathrm{nAChRs)} \mathrm{[43].} \mathrm{The} \mathrm{finding} \mathrm{of} \mathrm{human} \mathrm{neo-}$ cortical and rat striatal glutamatergic axon terminals expressing $\mathrm{nAChRs}$, thus mediating glutamate release, offers further possibilities of modulating glutamate excitotoxicity by KYNA [44]. However, other data argue that the compound lacks nAChR-inhibiting ability $[45,46]$. The controversial result might be due to the differences in research methods used. Nonetheless, when considering potential therapeutic interventions, elucidating the mechanism and effect of KNYA on $\mathrm{nAChRs}$ is of great importance and highly relevant [43]. Furthermore, the free-radical scavenger and antioxidant features of KYNA further extend its therapeutic potential [47]. 3-HK and 3-HAA are known free radical generators, thus contribute to oxidative stress and neurodegeneration [48]. However, there are reports which show these molecules have antioxidant properties as well. Due to the amino and hydroxyl aromatic group, they can be electron donors. Whether the proor the antioxidant properties will dominate depends on the activity of the redox system in the cell. In the presence of copper, 3-HK and 3-HAA generate superoxide anion and hydrogen peroxide, which then lead to highly reactive and cell-damaging quinonimine products [49]. QUIN is another neuroactive metabolite of the $\mathrm{KP}$, and it is a competitive NMDA receptor agonist that, as mentioned above, is proposed to be a key feature of HD excitotoxicity [50]. Accordingly, imbalance of the KP has been reported in several neurodegenerative diseases such as Parkinson's disease (PD), AD and HD (reviewed in [51]). Targeting specific enzymes of the pathway seems to be a feasible way of amending the imbalance between neuroprotectant and neurotoxic compounds, and by this prevent, or slowdown neurodegeneration.

\subsection{Kynurenine pathway alterations in Huntington's disease}

In light of the wide range of neuroactive kynurenines with their various effects on neuron viability and function, it is not surprising that the involvement of the pathway in HD has gathered a lot of interest in the past decades. In the following section, we will discuss some of the findings on the relationship between 


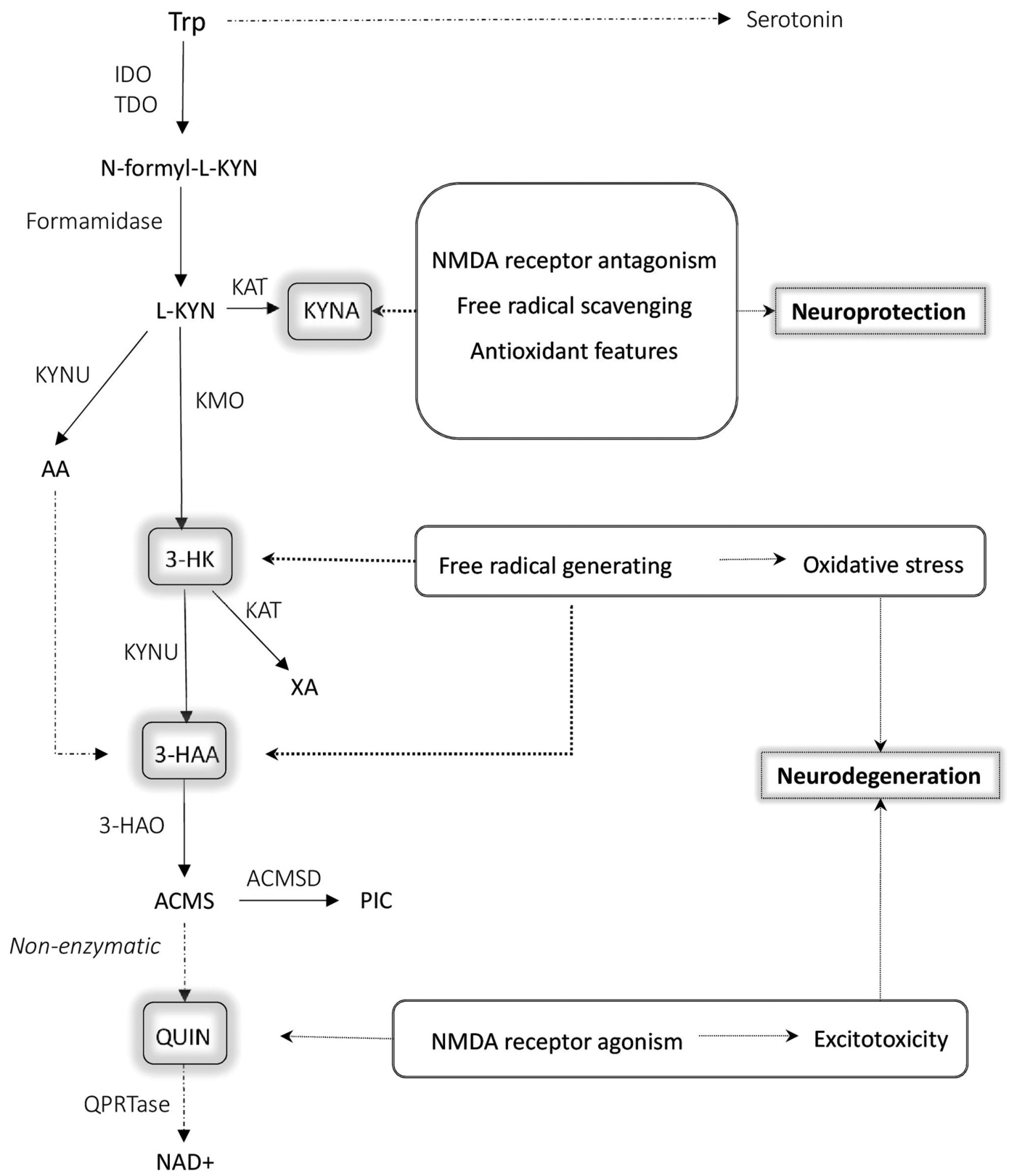

Figure 1. The kynurenine pathway with its neuroactive metabolites highlighted.

Among the KP metabolites KYNA, 3-HK and 3-HAA are known to have neuroactive properties. KYNA, due to its NMDA receptor antagonism, antioxidant and free radical scavenging features, is a neuroprotective compound. On the contrary, 3-HK and 3-HAA can cause excessive ROS production, while QUIN is a known NMDA receptor agonist, therefore a disproportionate level of these metabolites can lead to neurodegeneration.

Abbreviations: ROS: reactive oxygen species; Trp: tryptophan; IDO1,2: indoleamine 2,3-dioxygenase; TDO: tryptophan-2,3-dioxygenase; KYN: kynurenine; KAT: kynurenine aminotransferase; KYNA: kynurenic acid; KYNU: Kynureninase; AA: Anthranilic acid; KMO: Kynurenine-3-monooxygenase; 3-HK: 3-hydroxy-L-kynurenine; XA: Xanthurenic acid; 3-HAA: 3-hydroxyanthranilic acid; 3-HAO: 3-hydroxyanthranilate-3,4-dioxygenase; ACMS: 2-amino-3-carboxymuconate-semialdehide; ACMSD: Aminocarboxymuconate-semialdehyde-decarboxylase; PIC: Picolinic acid; QUIN: quinolinic acid; QPRTase: quinolate phospho-ribosyltransferase; NAD+: Nicotinamide adenine dinucleotide, NMDA: N-methyl-D-aspartate

the KP and HD. Tables 1 and 2 summarize changes in enzyme activity and kynurenine metabolite levels detected in HD patients and/or in animal models of the disease.

The KP is proposed to contribute to the development and progression of HD by several mechanisms. Here we consider those which are linked directly to KP functions. On one hand, the imbalance in the production of NMDA receptor agonist (QUIN) and antagonist (KYNA) compounds can lead to excititoxicity via NMDA receptor overactivation. On the other hand, excessive amounts of $3-\mathrm{HK}$ and $3-\mathrm{HAA}$ can lead to ROS accumulation thus causing oxidative stress that leads to neurodegeneration [20]. Furthermore, the activity of KP might greatly affect neurotransmitter production thus neuropsychiatric symptoms of the disease.

Mutant HTT can be one of the culprits of KP upregulation and the consequent rise in 3-HK and QUIN levels leading to ROS toxicity. It has been proposed that cytokines secreted by mutant $\mathrm{HTT}$-expressing dysfunctional neurons induce microglial activation [52]. The upregulated state of $\mathrm{KP}$ in activated microglia results in elevated levels of 3-HK and QUIN, leading to neuronal 
damage [52]. In another scenario, mutant HTT protein expressed directly in astrocytes and microglia leads to KP upregulation and resultant ROS overproduction [53].

The upregulated state of the pathway in the disorder was revealed by a study of Stoy and colleagues, who determined different KP metabolite levels in blood samples of HD patients [20]. The levels of KYN in HD patient samples were found to be double that of controls, suggesting an enhanced Trp to KYN conversion due to the increased activity of either IDO and/or TDO. This assumption is in accordance with the elevated KYN/Trp ratio (an indicator of IDO activity) found in HD patients [20], as no difference was observed between patients and controls either in Trp or KYNA levels. The decreased KYNA/KYN ratio resulting from elevated KYN and unchanged KYNA levels suggests a lower KAT activity in $\mathrm{HD}$. Besides KYN, a significant increase was observed in the amount of XA in HD patients, whereas their levels with

Table 1. Reported activity changes of kynurenine pathway enzymes that affect Huntington's disease.

\begin{tabular}{lcllc}
\hline Enzyme & $\begin{array}{c}\text { Change in } \\
\text { activity }\end{array}$ & \multicolumn{1}{c}{ Species } & \multicolumn{1}{c}{ Sample } & Reference \\
\hline IDO & $\uparrow$ & Human & blood & {$[20]$} \\
KYNU & $\downarrow$ & Mouse (R6/2) & brain & {$[64]$} \\
KAT & $\downarrow ?$ & Human & striatum & {$[54]$} \\
& $\downarrow$ & Human & striatum & {$[55]$} \\
& $\downarrow ?$ & Human & blood & {$[20]$} \\
KMO & $\uparrow$ & Mouse (R6/2) & brain & {$[64]$} \\
$3-H A O$ & $\uparrow$ & Human & brain (most & {$[57]$} \\
& & & prominently in & \\
& & & striatum) & \\
\hline
\end{tabular}

Abbreviations: IDO: indoleamine 2,3-dioxygenase; KYNU: kynureninase; KAT: kynurenine aminotransferase; KMO: kynurenine monooxygenase; 3-HAO: 3-hydroxyanthranilate-3,4-dioxygenase; $=:$ no detectable change; $\uparrow:$ increase; $\downarrow$ : decrease respect to the free radical generators 3-HAA and 3-HK were significantly decreased. Blood levels of QUIN showed large variations, both in patient and control study groups, not revealing significant differences between them [20].

An upregulated state of the pathway was also found in animal models of the disease. A significant decrease was found in the levels of Trp, accompanied with an increase in the KYN/Trp ratio in the brain of the 3-nitropropionic acid (3-NP) toxin mouse model [13]. Besides Trp, a decrease in cerebellar 3-HK levels was also observed [13].

Though analysis of blood samples did not reveal any change, a decrease in the level of the neuroprotective KYNA has been reported in HD postmortem cerebral cortex [14], putamen [54], nucleus caudatus [55], and CSF [54,56]. A decrease in levels of KYNA occurs simultaneously with an increase in the KYN/KYNA ratio, suggesting reduced KAT enzyme activity in HD brain [54], as was found in peripheral blood. Results of Jauch and colleagues strengthened this hypothesis, by finding significant decreases of KATI and II activities in the striatum samples of HD patients [55]. Interestingly, change in the enzyme activity was not found in other brain areas, suggesting the impairment of KYNA synthesis to be selective to the striatum [55].

Besides KATs, changes in the activity of another enzyme, 3-HAO has been reported in HD cases [57]. In contrast with KATs, 3-HAO activity was found to be increased in postmortem HD brain, most prominently in the striatum [57]. As astrocytes contain 3-HAO, it was proposed that the increase of enzyme activity might be partly due to astrogliosis, characteristic of HD striatum [57]. The involvement of astrocytes in HD pathogenesis and disease progression is multifaceted. Astrocytes lack

Table 2. Kynurenine metabolite level changes in Huntington's disease.

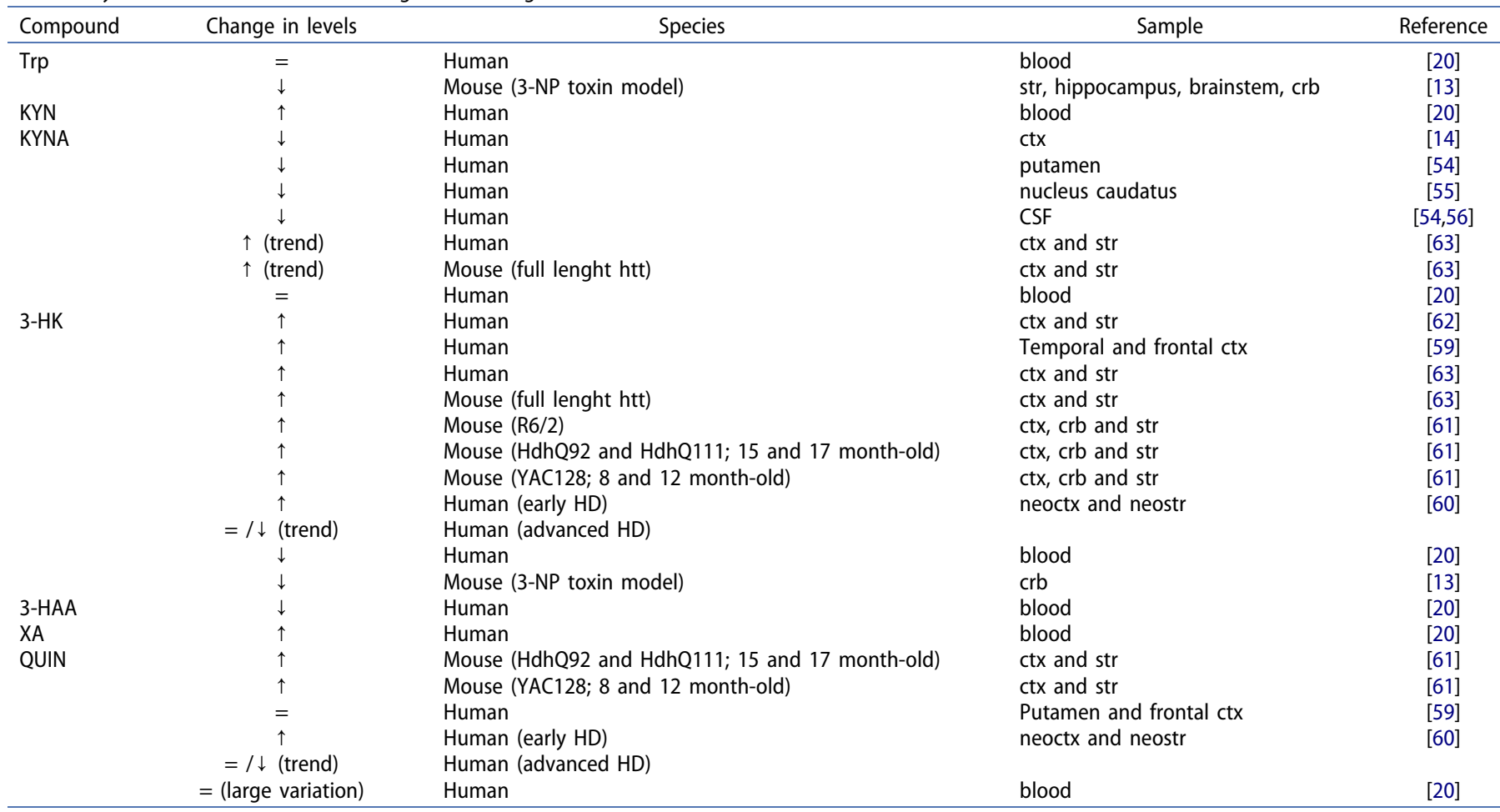

Abbreviations: Trp: tryptophan; 3-NP: 3-nitropropionic acid; KYN: kynurenine; KYNA: kynurenic acid; 3-HK: 3-hydroxy-L-kynurenine; 3-HAA: 3-hydroxyanthranilic acid; XA: xanthurenic acid; QUIN: quinolinic acid; = : no detectable change; $\uparrow$ : Increase; $\downarrow$ : Decrease; ctx: Cortex; CSF: Cerebrospinal fluid; str: Striatum; crb: Cerebellum; HD: Huntington's disease 
$350 \mathrm{KMO}$, therefore, while capable of KYNA and KYN formation, they only produce the down-stream neurotoxic metabolites such as QUIN and 3-HK in small amounts or not at all [58]. Moreover, astrocytes possess IFN $\gamma$-inducible quinolate phospho-ribosyltransferase (QPRTase) which degrades even the small amounts of QUIN produced from AA in astrocytes [58]. QPRTase, however, is easily saturated which can lead to QUIN accumulation. Increase in QUIN levels can also be the result of excessive KYN production, as it can be converted into the neurotoxic metabolite by the infiltrating macrophages and activated microglia [58]. Thus, under normal circumstances astroglia have neuroprotective effects due to their QUIN catabolizing and KYNA synthesizing ability, however, in the presence of activated microglia and macrophages astrocytes cause neuronal damage indirectly [58]. These findings raise the possibility of interfering QUIN-related excitotoxicity and consequent neurodegeneration by diminishing the formation of KYN in astrocytes via inhibition of the first enzyme of the pathway, IDO1.

The observation that HD symptoms can be modeled by 370 injection of QUIN into murine striatum predicted the compound would contribute to the disease in humans as well. Early findings by Reynolds and colleagues did not support this hypothesis [59], more recent results on the other hand are in accord with the idea, which assumes a role for QUIN in HD neurodegeneration: elevated levels of the NMDA receptor agonist have been found in both HD patients [60] and several animal models of the disease [61]. Further findings strongly suggest that QUIN, alongside 3-HK, has a role primarily in the early stages of the disease. Postmortem investigation of $\mathrm{HD}$ neocortex and neostriatum revealed that QUIN and 3-HK levels were elevated in early stages of the disease, however, with disease progression the levels of these metabolites seemed to normalize or even decrease [60].

Elevation in the level of 3-HK has been described repeatedly, both in postmortem human HD brain tissue and animal models [59-63]. Recent findings by Sathyasaikumar and colleagues indicate that an increase in 3-HK levels can result from either increased KMO or decreased KYNU activity, as these two enzymes are responsible for the synthesis and degradation of 3-HK, respectively [64]. However, it should be emphasized that approximately $60 \%$ of CNS resident L-KYN - that is the precursor of 3-HK - is transported through the BBB from the periphery [65]. Therefore, it cannot be overlooked that besides the combined effects of increased synthesis and reduced degradation levels, the accelerated influx of L-KYN is likely a result of increased L-KYN production in the periphery, which then also contributes to the elevated 3-HK levels in the CNS.

In a study, Guidetti et al. found that elevated 3-HK levels were accompanied by a parallel elevation of KYNA in the neostriatum of stage $1 \mathrm{HD}$ patients and in brain of a transgenic mouse HD model [63]. The increase of 3-HK levels was, however, more prominent, resulting in a high 3-HK/KYNA ratio [63]. As microglia possess the whole enzyme apparatus of the $\mathrm{KP}$, while, as mentioned above, astrocytes lack KMO, the marked elevation of 3-HK is presumably due to overactivated microglia. So this observation can serve as a further indication of the dysregulation in these cells in HD pathology [63]. Considering the above, one might propose that a more feasible way of intervening in the KP in HD is to decrease QUINrelated excitotoxicity by directly inhibiting the 3-HAA enzyme. However, blocking the pathway before the formation of QUIN could lead to the accumulation of other upstream metabolites, such as the free radical-generating, therefore potentially toxic, 3-HK and 3-HAA. This does not exclude 3-HAA as a target of therapeutic intervention though but highlights the complexity of interfering in the pathway.

KP metabolites might be used as new biomarkers for HD by monitoring the changes in their levels. An ongoing trial (HDClarity, ClinicalTrials.gov identifier: NCT02855476) aims to discover such novel biomarkers in the CSF, and uncover relationships between disease severity and KP metabolite levels. The study is expected to be completed by 2020 , and hopefully will provide important new information on potential therapeutic targets as well.

\subsection{Attempts to modulate elements of the kynurenine pathway as therapeutic targets for Huntington's disease}

Observations that imbalances of toxic and neuroprotective metabolites of the KP play roles in HD draw attention to elements of the pathway as potential therapeutic targets offering means to restore the balance (reviewed in [66]). This can be envisioned either by enzyme modulation, thus enhancing the production of the neuroprotectant KYNA or by utilizing its analog or alternatively its prodrug. The combined use of L-KYN and probenecid has been shown to have protective effects when considering both behavioral and neurochemical aspects in a QUIN-induced rat model of HD [67]. Similarly, beneficial effects have also been reported for the synthetic prodrug, 4-chlorokynurenyne (4-CL-KYN) in a QUIN and malonate-induced rat HD model [68]. 4-CL-KYN is the prodrug of 7-chlorokynurenynic acid (7-CL-KYNA), a selective glycin/ NMDA receptor antagonist. 4-CL-KYN, administered in intrastriatal infusion, was locally metabolized into 7-CL-KYNA and attenuated the toxic effects of both QUIN and malonate [68]. Lately, intraperitoneal injection of a novel KYNA analog, $\mathrm{N}$-(2-N-dimethylaminoethyl)-4-oxo-1 $\mathrm{H}$-quinoline-2-carboxamide hydrochloride was found to extend survival, improve motor functions, increase body weight, and prevent the loss of striatal neurons in a transgenic mouse model of the disease [69].

Among therapy approaches based on altering enzyme activities, targeting $\mathrm{KMO}$ has so far been in the spotlight [70]. The aim of such approaches is to enhance KYNA production by blocking the conversion of L-KYN to 3-HK. Elevation in brain KYNA levels can be achieved by intraventricular application of nicotinylalanine [71] and a novel peripheral KMO inhibitor, JM6, which is the prodrug of Ro 61-8048 [72]. Though neither JM6 nor Ro 61-8048 can cross the BBB, an elevation in the level of KYNA can be observed in the CNS even after oral administration of JM6. This is likely due to peripheral accumulation of the BBB penetrant KYN, which is metabolized into KYNA after entering the brain [72]. 
Based on the quinolate excitotoxicity-hypothesis, targeting NMDA receptors might also seem to be a reasonable therapeutic approach for HD. However, the feasibility of such efforts should be weighed with great precaution: in transgenic HD mice low-dose memantine treatment, which causes inhibition of extrasynaptic NMDA receptors, resulted in an improvement in the symptoms, while blockage of synaptic receptors by high-dose memantine had the opposite effects [73]. Synaptic NMDA receptor activity was protective against mutant $\mathrm{Htt}$ protein, while activation of extrasynaptic NMDA receptors decreased neuronal survival [73].

Similarly to NMDA receptor-modulating therapies, regulating KYNA levels also requires care as there is a growing body of evidence on the negative effects of elevated KYNA levels on cognitive functions under certain circumstances [74].

\section{Indoleamine 2,3-dioxygenase}

\subsection{Indoleamine 2,3-dioxygenase enzymes}

IDO is the first and rate-limiting enzyme of the KP catalyzing the conversion of Trp to $\mathrm{N}$-formyl-L-kynurenine (Figure 1). In humans, two IDO enzymes have been identified, which are referred to as IDO1 and IDO2. In papers published before the discovery of IDO2 in 2007 [75,76], the 'IDO' designation was used in general and some studies even nowadays use the term 'IDO' for IDO1. For clarity, we will use the IDO1 and IDO2 nomenclature, distinguishing the two enzymes whenever possible.

The genes encoding IDO1 and IDO2 are adjacent on chromosome 8 , suggesting their common origin [77]. The two enzymes have $43 \%$ amino acid identity, including residues critical for catalytic activity [75]. Both IDO can convert the same substrates (L- and D-Trp, tryptamine, 5-hydroxytyptamine, serotonin), IDO1 however, has higher catalytic activity [78]. In fact, the Michaelis Constant (Km) of IDO2 for Trp is over 100-fold higher than physiological Trp concentrations which makes the direct role of IDO2 in Trp degradation questionable and suggests another, so far unidentified natural substrate for the enzyme [79]. Available data suggest low levels of IDO2 expression in liver, testis, and thyroid [80]. In addition, frequent occurrence of mutant IDO2 alleles leads to non-functioning IDO2 in 50\% of the population [76].

More information is available regarding IDO1 activity, tissue-specific expression, and functions in the human body. Under physiological conditions, IDO1 expression can be detected in several human tissues, such as lymphoid organs, epithelial cells of the female genital tract, placenta, and lung parenchyma (for a review see [80]). IDO1 is also expressed in various immune cells, such as DCs, monocytes, MAs, and microglia [81]. Its expression can be stimulated by various immunomodulators, including IFNs, lipopolysaccharides (LPS), ILs (IL1 $\beta$, IL6), TNFs (TNFa) and prostaglandins (PGE2), of which the strongest inducer seems to be IFNY $[37,80]$. IDO1 has an important role in regulating the immune system and maintaining immune-homeostasis. It can exert its immunemodulating effects both by decreasing the amount of available Trp and by producing kynurenines (see below).

In short, despite structural similarities with IDO1, the low catalytic activity, low expression level and high frequency of non-functional variant forms make IDO2 less favorable, and IDO1 a more promising target for therapeutic approaches.

\subsection{The effects of indoleamine 2,3-dioxygenase 1 on immune functions}

It is beyond the scope of this review to provide a comprehensive discussion of the diverse functions of IDO1 and the affected mechanisms. Instead, in this section, we highlight the complexity of IDO1's contribution to immune regulation (Figure 2).

The first report on the role of IDO in immune responses was published by Munn and colleagues [82] who proposed that allogeneic fetal rejection in a mouse model was prevented by the T-cell modulating effect of placental cells expressing Ido. They hypothesized that by diminishing the level of available Trp, Ido inhibited maternal T-cell responses, which led to the loss of the allogeneic fetus [82]. The mechanism by which $T$ cell suppression is achieved has been argued recently and proposed to be due to the formation of kynurenine metabolites, rather than to Trp depletion [83]. Either way, it seems that IDO1 via Trp degradation or metabolite synthesis can exert immune modulating effects (for a review see [84]).

IDO1 also exhibits inhibitory effects on T effector cells ( $\left.T_{\text {efff }}\right)$, thus it has anti-inflammatory properties. One way $T_{\text {eff }}$ cells can be inhibited is indirectly, by promoting naive T-cell differentiation into regulatory $T$-cells $\left(T_{\text {reg }}\right)$ [85]. Kynurenine metabolites were also found to be able to stabilize the immunosuppressive phenotype of $T_{\text {reg }}$ cells, avoiding their transformation into proinflammatory $T$ helper-like effector cells at inflammation sites [86].

Direct inhibition of $\mathrm{T}_{\text {eff }}$ cells by IDO1 can be achieved by inducing clonal anergy [87], cell cycle arrest, and apoptosis [88]. Besides affecting $T_{\text {eff }}$ cell survival, the activation of IDO1 and consequent production of kynurenines results in cell cycle arrest and apoptosis of B and natural killer (NK) cells, as well [88].

Under certain circumstances, however, instead of repressing, IDO1 can stimulate B cell responses and cause the activation of autoreactive $B$ cells leading to autoimmune processes [89]. The Janus-faced regulatory role of IDO1 in immune modulation is well illustrated by its effects on MAs. On the one hand, activated MAs expressing IDO1 deplete the Trp supply in their microenvironment, in this way enhancing their sensitivity to LPSs. This leads to an increase in their IL-6 production, promoting inflammatory processes [90]. On the other hand, over-expression of IDO1 triggers MAs to differentiate into immunosuppressing $M 2$, rather than "effector" $M 1$, thus promoting an immune tolerant state [91]. This self-regulatory mechanism enables the avoidance of excessive immune response. In certain infectious states, the prevention of exaggerated immune response is reached via increased IDO1 expression of DCs. The augmented expression of the enzyme leads to the excessive formation of KYN, which induces apoptosis of neutrophil granulocytes (NGs) at the site of inflammation [92]. Due to the consequent decrease in IFNY production, the accumulation of further NGs is limited, thus restraining the local immune response [92].

By controlling the quantity of available Trp through the formation of kynurenine metabolites, IDO1 has indirect effects 


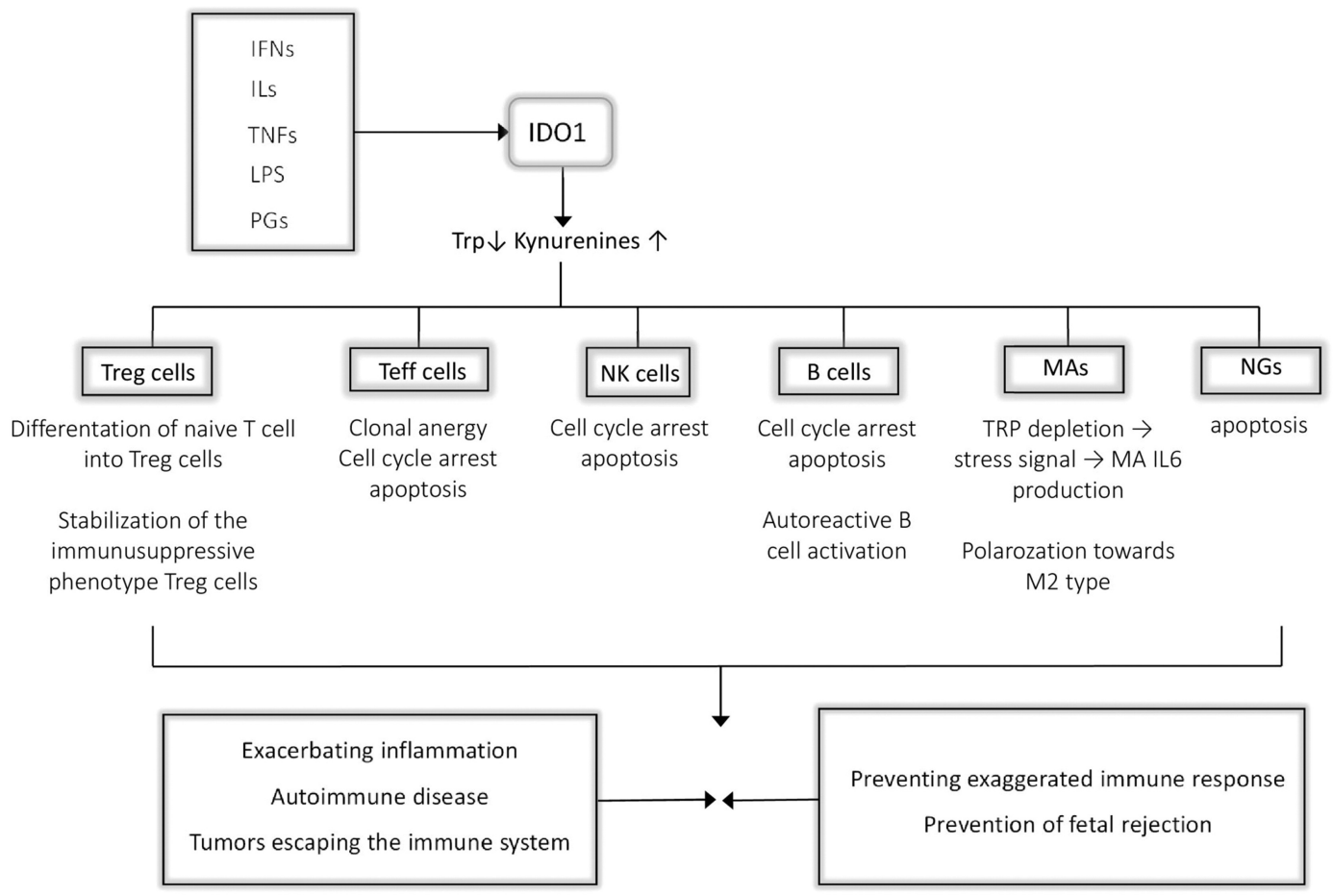

Figure 2. The immunomodulatory roles of indoleamine 2,3-dioxygenase.

IDO1 affects the activity of various immune cells, such as T and B cells, NK cells, macrophages, and neutrophile granulocytes via Trp depletion and the production of KP metabolites. Depending on the microenvironment and presence of further inflammatory molecules and immune cells, IDO1 can lead to both the progression of inflammatory processes and immune suppression.

Abbreviations: IFNs: Interferons; ILs: Interleukins; TNFs: Tumor necrosis factors; LPS: Lipopolysaccharide; PGs: Prostaglandines; Trp: Tryptophan; IDO1: Indoleamine 2,3-dioxygenase 1; Treg: Regulatory T-cell; Teff: Effector T-cell; NK cells: Natural killer cells; MAs: Macrophages; NGs: Neutrophil granulocytes.

on serotonin synthesis (Figure 1). In light of data on the involvement of the serotonin system in depression, targeting IDO1 also offers the possibility to manage mood alterations often present in HD.

In light of its diverse immune-modulating effects and its link with other metabolic pathways, not surprisingly, IDO1 has been implicated in several diseases in which changes in immunoregulation are observed. Besides fetal rejection [82] expression and/or function of IDO1 has been investigated and found to be altered in infectious $[93,94]$ and autoimmune diseases $[95,96]$, in the immune-escaping process of tumors [97] and in neurodegenerative diseases [98,99], such as HD.

\subsection{Indoleamine 2,3-dioxygenase as a potential therapeutic target for Huntington's disease treatment}

Despite being the rate-limiting enzyme in a pathway that converts Trp to neuroactive compounds, and having widespread function in immune modulation, data on IDO1 in respect of its role in HD are scarce. Nonetheless, information from both animal models and analysis of human tissues strongly suggests the involvement of the enzyme in the disease, making it worthy of attention in the search for new therapeutic targets for HD.

The observation that Ido1 expression is higher in wild-type mouse striatum compared to other brain regions, might serve as an indication of the role of IDO1 in HD [9]. Ido1 activity can expression [9]. Considering that MSNs are primarily prone to neurodegeneration in HD and that astrogliosis is a hallmark of the disease, these findings raise intriguing questions regarding the role of IDO1 in HD.

Despite the loss of cells that proposedly contribute most to IDO1 expression in the striatum, elevated Ido1 activity could be detected in the striatum of YAC128 mice, a model of the disease, resulting in increased levels of neurotoxic 3-HK production [9]. Increased enzyme activity was also reported in the blood of HD patients [20]. The decrease in the level of KYN after Trp depletion was found to be less prominent in HD patients compared to healthy controls, suggesting that the activity of IDO1 is higher in $\mathrm{HD}$ even during at shortage of its metabolite [20].

These findings point to the possibility of IDO1 inhibition as a therapeutic approach in HD. Results of studies conducted using fruit fly and murine models support the rationale of such an approach. In a Drosophila melanogaster HD model, genetic inhibition of the Tdo2 enzyme was found to be protective against mutant $\mathrm{Htt}$ protein neurotoxicity [100]. Drosophila possesses neither Ido1 nor Ido2 therefore Tdo2 is the first, rate-limiting enzyme of the pathway, which converts Trp to KYN [100]. The inhibition of Tdo2 shifts the pathway toward the formation of KYNA, thus enhancing neuroprotection [100]. One might propose that inhibition of IDO1 - as a functional analog of TDO2 - could have a similar effect both in higher model organisms and in humans, therefore, it might serve as an alternate way to improve neuroprotection.

Supporting this concept, it was found that in the absence of Ido1 expression in the striatum of Ido1 knockout (Ido-/-) 
mice, neurons in the striatum became less sensitive to NMDA receptor-mediated excitotoxicity, as it was demonstrated by the reduction of QUIN-induced neurotoxicity [101]. This can be partly explained by the significantly decreased levels of 3-HK observed in striatum of Ido-/- mice, as 3-HK has been shown to potentiate QUIN toxicity [102]. Interestingly, the lack of the enzyme did not cause any change in the levels of QUIN and KYNA [101].

Findings of Forrest and colleagues further strengthen the involvement of IDO1 activity in the progression of HD. Prominently lower levels of Trp and higher Trp/KYN ratios (indicating higher activity of IDO1 enzyme) were observed in most severely affected HD patients compared to patients with less severe clinical symptoms. The results of negative correlation between the number of CAG repeats and Trp levels, while positive correlation between CAG repeat number and KYN/Trp ratio suggests a link between disease severity and IDO1 activation $[34,103]$.

\section{Conclusion}

650 Overall, research focusing on IDO1 has uncovered several effects of the enzyme on pathways altered in HD. These data support the consideration of IDO1 as a therapeutic target for the disease and strongly warrant further studies exploring additional details of the processes affected. Findings accumulated so far strongly suggest that inhibition of the first step of the KP, such as decreased IDO1 activity, can be beneficial in preventing neuron loss. A supporting argument for targeting IDO1 can also be that by altering the balance of kynurenine metabolites via modulation of the activity of this enzyme, both the direct effects of neuroprotective compounds of the KP can be exploited, and indirectly, changes can be triggered in ROS production and in immune responses that are suggested to play roles in HD, as indicated by the consistent elevation of inflammatory markers in HD blood samples [20].

Last, but not least, through inhibition of IDO1, the metabolism of Trp can be shifted toward the synthesis of serotonin; thus, inhibition of IDO1 might also serve as a therapeutic invention against depressive symptoms in HD. Nonetheless, to our knowledge, no therapeutic interventions targeting the IDO enzyme with the intention for HD therapy have yet been tested.

\section{Expert opinion}

Identification of possible therapeutic targets in HD is urgent, as despite the severe motor, psychiatric and cognitive symptoms, the available treatment is mainly symptomatic. Recently HTT gene expression modulation by antisense oligonucleotide (ASO) has been presented as a promising therapeutic approach for HD treatment (IONIS-HTTRx; ClinicalTrials.gov identifier: NCT02519036; reviewed in [104]). A phase 1/2a regarding both tolerability and effectiveness, demonstrating the possibility of the ASO approach in HD treatment [105]. Gene expression modulating therapies, however, have several limitations (mode of implementation, age of the patients,

existing comorbidities, etc.), thus discovering other therapeutic methods is still clearly warranted.

Imbalances of the KP have been reported in HD on several occasions. Overproduction of QUIN leads to NMDA receptormediated excitotoxicity, a well-documented mechanism in HD pathogenesis. Elevated $3-\mathrm{HK}$ production contributes to augmented oxidative stress and consequent mitochondrial damage, and potentiates QUIN-induced toxicity of neurons. Consequently, modulating the activity of KP enzymes involved in the production and degradation of these metabolites might offer a possibility for therapeutic intervention. IDO1 catalyzes the first reaction of the KP and is the rate-limiting enzyme of the metabolic route. By modulating IDO1 activity, the balance of kynurenine metabolites could be shifted toward the production of neuroprotectant, rather than neurotoxic compounds.

Reflecting the neurodegeneration and consequent removal of degenerating cells, elevated inflammatory markers in HD patients strongly suggests a persistent state of inflammation. In light of the fact that IDO1 also plays a pivotal role in regulating immune mechanisms, the enzyme could be a potential target for the modulation of inflammatory processes related to HD as well.

IDO1 inhibition might also be beneficial in ameliorating depressive symptoms associated with the disease, as various abnormalities of the serotoninergic system have been linked to depression, among these, change in the Trp level directly affected by IDO1 [106]. By inhibition of ID01, Trp utilization via the KP is inhibited, thus higher level of amino acid is available as precursors for serotonin synthesis. The notion regarding the role of IDO1 in the development of depression is supported by the finding that peripheral inflammation, which is an inducer of IDO1, was shown to contribute to the development of depressive symptoms [107].

Despite the increasing body of evidence thus far accumulated that suggests dysregulation of $\mathrm{KP}$ in $\mathrm{HD}$ and data demonstrating the beneficial effects of IDO1 inhibition, no therapeutic intervention targeting the enzyme has been proposed for HD treatment. This might be due to the very complex nature of HD pathogenesis: even though several pathological changes have been proven or suggested to contribute to the disease, exploration of the exact mechanisms of pathogenesis requires further elucidation. The functional consequences of IDO1 activity are also diverse and finely tuned (e.g. self-defense against overactivated immune response versus exaggerated immunotolerance leading to autoimmunity); therefore, the elucidation of its precise effects under specific conditions poses formidable challenges. To explore the various possibilities and identify causal connections, which might further underline reasons to consider IDO1 as a therapeutic target for HD, extension of our knowledge on the variation in enzyme activity in the disease state and the contribution of that to HD is highly warranted.

However, inhibition of the enzyme can have side effects due to its complex role in immune regulation. At the site of inflammation, Th1 cells producing IFN $\gamma$ and other cytokines induce the expression of IDO1, causing the decrease of locally available Trp and increasing the formation of KP metabolites. 
These changes lead to the decrease of effector T-cell responses, thus preventing exaggerated immune responses, suggesting that IDO is a component of a self-regulatory, negative feedback loop. It can easily be conceived that disturbance to this finely tuned self-regulatory mechanism either by pharmacological enzyme inhibition or by genetically determined decreased enzyme activity - can lead to unwanted and excessive immune response. Such an effect was demonstrated in a mouse model of colitis, in which inhibition of the enzyme resulted in a higher mortality [108]. An exaggerated immune response might also lead to flair ups of autoimmune diseases, as was demonstrated in a proteolipid protein (PLP)-induced experimental autoimmune encephalomyelitis (EAE) mouse model of multiple sclerosis [96]. During inflammation in the CNS, higher IDO activity was measured, showing correlation to the clinical onset and severity of the disease [96]. It was found that treatment with the IDO inhibitor 1-Mt of EAE animals caused the exacerbation of the disease as demonstrated by the significantly higher clinical scores of the 1-Mt-treated group compared to untreated animals [96]. Precautions are warranted in enzyme inhibition, not only in the case of inflammatory processes but in pregnancy and tissue transplantant as well. Diminished immune tolerance in the placenta can result in the loss of allogeneic concepti as demonstrated by Munn and colleagues using the 1-Mt treated mouse model [82]. Furthermore, Ido-/- mice receiving bone marrow transplants showed a more severe course of graftversus-host colitis compared to the wild-type recipients [109].

Besides side effects, the selectivity of IDO1 inhibitors is another cardinal question that should not be overlooked. Some of the monoamine oxidase (MAO) inhibitors were found to inhibit IDO1. One cannot exclude the possibility of inhibitors of IDO1 affecting other enzymes as well [110].

Considering the above mentioned, one might propose that a more feasible way of intervening the KP in HD is to decrease QUIN-related excitotoxicity by directly inhibiting the 3-HAA enzyme. However, blocking the pathway before the formation of QUIN could lead to the accumulation of other upstream metabolites, such as the free radical-generating, therefore potentially toxic, 3-HK and 3-HAA. This does not exclude 3-HAA as a target of therapeutic intervention but highlights the complexity of interfering in the pathway.

A possible way to avoid the above-mentioned side effects of IDO inhibition is careful selection of the patients who receive such enzyme therapy. Application of IDO inhibitors in individuals suffering from autoimmune disease or are subjects of organ or tissue transplant should be carried out with great precaution and need monitoring of inflammatory processes.

Although inhibitors of IDO1 developed for and tested specifically in HD are nonexistent, IDO1-inhibiting compounds are under trial as promising drugs for cancer therapy, as decreasing the enzyme's activity can help to avoid immune surveillance of malignant tumors [111]. Out of the compounds tested in this respect, two have already reached clinical trial phase [111]. These are 1-methylD-tryptophan (1-D-MT), which was the first compound found to be potent in the micromolar range, and INCB24360, a hydroxyamidine derivate. Though the number of specific IDO1 inhibitors so far developed is scarce, hopefully, drug research will be able to develop other compounds for cancer and other diseases. Results of cancer research involving IDO1 activity modulation carry hope for therapeutic developments for HD as well. Compounds that have been proven to be safe based on trials in one area of medicine, hold great advantages for trials in other areas, improving time and cost efficiency, regardless of whether they have been effective with respect to the primary purpose of their development.

All things considered, IDO1 might serve as a valuable novel therapeutic target for HD as it offers possibilities for beneficially modulating neurodegeneration, inflammation, and serotoninerg neurotransmission simultaneously. As these complex processes are all affected in HD, IDO1 is clearly an unexplored target in the search for new approaches to HD therapy. Ongoing trials aimed at recognizing KP metabolites as biomarkers for HD and the thus far developed IDO1 inhibitors under clinical trial justify further therapeutic developments.

\section{Funding}

This paper was not funded. The current work was supported by Economic Development and Innovation Operational Programme - GINOP-2.3.2-152016-00034, Hungarian Brain Research Program - Grant No. 20171.2.1-NKP-2017-00002 NAP VI/4, Ministry of Human Capacities, Hungary grant 20391-3/2018/FEKUSTRAT and the MTA-SZTE Neuroscience Research Group of the Hungarian Academy of Sciences and the University of Szeged.

\section{Declaration of interest}

The authors have no relevant affiliations or financial involvement with any organization or entity with a financial interest in or financial conflict with the subject matter or materials discussed in the manuscript. This includes employment, consultancies, honoraria, stock ownership or options, expert testimony, grants or patents received or pending, or royalties.

\section{Reviewer disclosures}

Peer reviewers on this manuscript have no relevant financial or other relationships to disclose

\section{References}

Papers of special note have been highlighted as either of interest $(\bullet)$ or of considerable interest (••) to readers.

1. Novak MJU, Tabrizi SJ. Clinical review Huntington's disease. BMJ. 2010;340:34-40.

2. Baig SS, Strong M, Quarrell OWJ. The global prevalence of Huntington's disease: a systematic review and discussion. Neurodegener Dis Manag. 2016;6:331-343.

3. Macdonald ME, Ambrose CM, Duyao MP, et al. A novel gene containing a trinucleotide that is expanded and unstable on Huntington's disease chromosomes. Cell. 1993;72:971-983.

4. Zuccato C, Valenza M, Cattaneo E. Molecular mechanisms and potential therapeutical targets in Huntington's disease. Physiol Rev. 2010;10:905-981.

5. Sorolla MA, Rodríguez-Colman MJ, Vall-Llaura $\mathrm{N}$, et al. Protein oxidation in Huntington disease. BioFactors. 2012;38:173-185.

6. Perutz MF. Glutamine repeats and neurodegenerative diseases: molecular aspects. Trends Biochem Sci. 1999;24:58-63. 
7. Kuemmerle S, Gutekunst C, Klein AM, et al. Huntingtin aggregates may not predict neuronal death in Huntington's Ddsease. Ann Neurol. 1999;46:842-849.

8. Arrasate M, Mitra S, Schweitzer ES, et al. Inclusion body formation reduces levels of mutant huntingtin and the risk of neuronal death. Nature. 2004;431:805-810.

9. Mazarei G, Budac DP, Lu G, et al. Age-dependent alterations of the kynurenine pathway in the YAC128 mouse model of Huntington disease. J Neurochem. 2013;127:852-867.

865 10. Ehrlich ME. Huntington's disease and the striatal medium spiny neuron: cell-autonomous and non-cell-autonomous mechanisms of disease. Neurotherapeutics. 2012;9:270-284.

11. Yager LM, Garcia AF, Wunsch AM, et al. The ins and outs of the striatum: role in drug addiction. Neuroscience. 2015;301:529-541.

870 12. Benarroch EE. Effects of acetylcholine in the striatum: recent insights and therapeutic implications. Neurology. 2012;79:247-281.

13. Veres $G$, Molnár M, Zádori $D$, et al. Central nervous system-specific alterations in the tryptophan metabolism in the 3-nitropropionic acid model of Huntington's disease. Pharmacol Biochem Behav. 2015;132:115-124.

14. Beal MF, Matson WR, Storey E, et al. Kynurenic acid concentrations are reduced in Huntington's disease cerebral cortex. J Neurol Sci. 1992;108:80-87.

15. Coyle JT, Schwarcz R. Lesion of striatal neurones with kainic acid provides a model for Huntington's chorea. Nature. 1976;263:244-246.

16. Beal MF, Kowall NW, Ellison DW, et al. Replication of the neurochemical characteristics of Huntington's disease by quinolinic acid. Nature. 1986;321:168-171.

885 .• The work of Beal and collegues presents the QUIN- model of HD.

17. Stone TW, Perkins MN. Quinolinic acid: a potent endogenous excitant at amino acid receptors in CNS. Eur J Pharmacol. 1981;72:411-412.

18. Young AB, Greenamyre JT, Hollingsworth $Z$, et al. NMDA receptor losses in putamen from patients with Huntington's disease. Science. 1988;241:981-983.

19. Albin RL, Young $A B$, Penney $J B$, et al. Abnormalities of striatal projection neurons and $\mathrm{N}$-methyl-D-aspartate receptors in presymptomatic Huntington's disease. N Engl J Med. 1990;322:1293-1298.

20. Stoy N, Mackay GM, Forrest CM, et al. Tryptophan metabolism and oxidative stress in patients with Huntington's disease. J Neurochem. 2005;93:611-623.

.. In this work, Stoy and collegues indicate that HD is accompanied by elevated inflammatory markers - not only in the CNS, but in the periphery as well.

21. Zádori D, Geisz A, Vámos E, et al. Valproate ameliorates the survival and the motor performance in a transgenic mouse model of Huntington's disease. Pharmacol Biochem Behav. 2009;94:148-153.

905 22. Stahl WL, Swanson PD. Biochemical abnormalities in Huntington's chorea brains. Neurology. 1974;24:813-819.

23. Greene JG, Porter RHP, Eller RV, et al. Inhibition of succinate dehydrogenase by malonic acid produces an "excitotoxic" lesion in rat striatum. J Neurochem. 1993;61:1151-1154.

910 24. Jenkins BG, Koroshetz WJ, Beal MF, et al. Evidence for impairment of energy metabolism in vivo in Huntington's disease using localized 1H NMR spectroscopy. Neurology. 1993;43:2689-2695.

25. Koroshetz WJ, Jenkins BG, Rosen BR, et al. Energy metabolism defects in Huntington's disease and effects of coenzyme Q10. Ann Neurol. 1997;41:160-165.

26. Schilling G, Coonfield ML, Ross CA, et al. Coenzyme Q10 and remacemide hydrochloride ameliorate motor deficits in a Huntington's disease transgenic mouse model. Neurosci Lett. 2001;315:149-153.

27. Ferrante RJ, Andreassen OA, Dedeoglu A, et al. Therapeutic effects of Coenzyme Q10 and remacemide in transgenic mouse models of Huntington's disease. J Neurosci. 2002;22:1592-1599.
28. Group THS. A randomized, placebo-controlled trial of coenzyme Q10 and remacemide in Huntington's disease. Neurology. 2001;57:397-404.

29. Wyttenbach A, Sauvageot O, Carmichael J, et al. Heat shock protein 27 prevents cellular polyglutamine toxicity and suppresses the increase of reactive oxygen species caused by huntingtin. Hum Mol Genet. 2002;11:1137-1151.

30. Tabrizi SJ, Cleeter MWJ, Xuereb J, et al. Biochemical abnormalities and excitotoxicity in Huntington's disease brain. Ann Neurol. 1999;45:25-32.

31. Sapp E, Kegel KB, Aronin N, et al. Early and progressive accumulation of reactive microglia in the Huntington disease brain. J Neuropathol Exp Neurol. 2001;60:161-172.

32. Simmons DA, Casale M, Alcon B, et al. Ferritin accumulation in dystrophic microglia is an early event in the development of Huntington's disease. Glia. 2007;55:1074-1084.

33. Singhrao SK, Neal JW, Morgan BP, et al. Increased complement biosynthesis by microglia and complement activation on neurons in Huntington's disease. Exp Neurol. 1999;159:362-376.

34. Forrest CM, Mackay GM, Stoy N, et al. Blood levels of kynurenines, interleukin-23 and soluble human leucocyte antigen- $G$ at different stages of Huntington's disease. J Neurochem. 2010;112:112-122.

35. Paulsen JS, Nehl C, Hoth KF, et al. Depression and stages of Huntington's disease. J Neuropsychiatry Clin Neurosci. 2005; 17:496-502.

36. Leklem JE. Quantitative aspects of tryptophan metabolism in humans and other species: a review. Am J Clin Nutr. 1971;24:659-672.

37. Mándi $Y$, Vécsei L. The kynurenine system and immunoregulation. J Neural Transm. 2012;119:197-209.

38. Pardridge WM. Blood-brain barrier carrier-mediated transport and brain metabolism of amino acids. Neurochem Res. 1998;23:635-644.

39. Wolf $\mathrm{H}$. The effect of hormones and vitamin $\mathrm{B} 6$ on urinary excretion of metabolites of the kynurenine pathway. Scand J Clin Lab Suppl. 1974;136:1-186.

40. Han Q, Cai T, Tagle DA, et al. Structure, expression, and function of kynurenine aminotransferases in human and rodent brains. Cell Mol Life Sci. 2010;67:353-368.

41. Okuno $E$, Nakamura $M$, Schwarcz R. Two kynurenine aminotransferases in human brain. Brain Res. 1991;542:307-312.

42. Elmslie KS, Yoshikami D. Effects of kynurenate on root potentials evoked by synaptic activity and amino acids in the frog spinal cord. Brain Res. 1985;330:265-272.

43. Albuquerque EX, Schwarcz R. Kynurenic acid as an antagonist of a7nicotinic Acetylcholine receptors in the brain: facts and challenges. Biochem Pharmacol. 2013;85:1027-1032.

44. Marchi M, Risso F, Viola C, et al. Direct evidence that release-stimulating alpha-7nicotinic cholinergic receptors are localized on human and rat brain glutamatergic axon terminals. J Neurochem. 2002;80:1071-1078.

45. Mok MHS, Fricker A-C, Weil A, et al. Electrophysiological characterisation of the actions of kynurenic acid at ligand-gated ion channels. Neuropharmacology. 2009;57:242-249.

46. Dobelis P, Staley KJ, Cooper DC. Lack of modulation of nicotinic acetylcholine alpha-7 receptor currents by kynurenic acid in adult hippocampal interneurons. PLoS. 2012;7:1-6.

47. Lugo-Huitrón $R$, Blanco-Ayala $T$, Ugalde-Muñiz $P$, et al. Neurotoxicology and teratology on the antioxidant properties of kynurenic acid: free radical scavenging activity and inhibition of oxidative stress. Neurotoxicol Teratol. 2011;33:538-547.

48. Shoki O, Nobuyoshi N, Hiroshi S, et al. 3-hydroxykynurenine, an endogenous oxidative stress generator, causes neuronal cell death with apoptotic features and region selectivity. J Neurochem [Internet]. 1998;70:299-307.

49. Zhuravlev AV, Zakharov GA, Shchegolev BF, et al. Antioxidant properties of kynurenines: density functional theory calculations. PLOS Compotational Biol [Internet]. 2016;1-31. DOI:10.1371/journal.pcbi.1005213 
50. Guillemin GJ. Quinolinic acid, the inescapable neurotoxin. FEBS J. 2012;279:1356-1365.

51. Vecsei L, Szalardy L, Fulop F, et al. Kynurenines in the CNS: recent advances and new questions. Nat Rev Drug Discov. 2012 [cited Dec 15];12:64-82.

995 52. Schwarcz R, Pellicciari R. Manipulation of brain kynurenines: glial targets, neuronal effects, and clinical opportunities. J Pharmacol Exp Ther. 2002;303:1-10.

- This article reveals the link between mutant HTT and the KP.

53. Giorgini F, Guidetti P, Nguyen Q, et al. A genomic screen in yeast implicates kynurenine 3- monooxygenase as a therapeutic target for Huntington's disease. Nat Genet. 2005;37:526-531.

54. Beal MF, Matson WR, Swartz KJ, et al. Kynurenine pathway measurements in Huntington's disease striatum: evidence for reduced formation of kynurenic acid. J Neurochem. 1990;55:1327-1339.

1005 55. Jauch D, Urbanska EM, Guidetti P, et al. Dysfunction of brain kynurenic acid metabolism in Huntington's disease: focus on kynurenine aminotransferases. J Neurol Sci. 1995;130:39-47.

56. Heyes MP, Saito K, Crowley JS, et al. Quinolinic acid and kynurenine pathway metabolism in inflammatory and non-inflammatory neurological disease. Brain. 1992;115:1249-1273.

57. Schwarcz R, Okuno E, White RJ, et al. 3-Hydroxyanthranilate oxygenase activity is increased in the brains of Huntington disease victims. Proc Natl Acad Sci USA 1988;85:4079-4081.

58. Guillemin GJ, Kerr SJ, Smythe GA, et al. Kynurenine pathway metabolism in human astrocytes: a paradox for neuronal protection. J Neurochem. 2001;78:842-853.

59. Reynolds GP, Pearson SJ, Halket J, et al. Brain quinolinic acid in Huntington's disease. J Neurochem. 1988;50:1959-1960.

60. Guidetti P, Luthi-Carter RE, Augood SJ, et al. Neostriatal and cortical quinolinate levels are increased in early grade Huntington's disease. Neurobiol Dis. 2004;17:455-461.

61. Guidetti P, Bates GP, Graham RK, et al. Elevated brain 3-hydroxykynurenine and quinolinate levels in Huntington disease mice. Neurob. 2006;23:190-197.

1025 62. Pearson SJ, Reynolds GP. Increased brain concentrations of a neurotoxin, 3-hydroxykynurenine, in Huntington's disease. Neurosci Lett. 1992;144:199-201.

63. Guidetti P, Reddy PH, Tagle DA, et al. Early kynurenergic impairment in Huntington's disease and in a transgenic animal model. Neurosci Lett. 2000;283:233-235.

64. Sathyasaikumar KV, Stachowski EK, Amoria L, et al. Dysfunctional kynurenine pathway metabolism in the $R 6 / 2$ mouse model of Huntington's disease. J Neuro. 2010;113:1416-1425.

65. Gál EM, Sherman AD. Synthesis and metabolism of L-kynurenine in rat brain. J Neurochem. 1978;30:607-613.

66. Vamos E, Pardutz A, Klivenyi $P$, et al. The role of kynurenines in disorders of the central nervous system: possibilities for neuroprotection. J Neurol Sci. 2009;283:21-27.

67. Santamaria A, Rios C, Solís-Hernández F, et al. Systemic DL-kynurenine and probenecid pretreatment attenuates quinolinic acid-induced neurotoxicity in rats. Neuropharmacology. 1996;35:23-28.

68. Guidetti P, Wu H, Schwarcz R. In situ produced 7-chlorokynurenate provides protection against quinolinate- and malonate-induced neurotoxicity in the rat striatum. Exp Neurol. 2000;163:123-130.

69. Zádori D, Nyiri G, Szőnyi A, et al. Neuroprotective effects of a novel kynurenic acid analogue in a transgenic mouse model of Huntington's disease. J Neural Transm. 2011;118:865-875.

70. Toledo-Sherman LM, Prime ME, Mrzljak L, et al. Development of a series of aryl pyrimidine kynurenine monooxygenase inhibitors as potential therapeutic agents for the treatment of Huntingtons disease. J Med Chem. 2015;58:1159-1183.

71. Harris CA, Miranda AF, Tanguay JJ, et al. Modulation of striatal quinolinate neurotoxicity by elevation of endogenous brain kynurenic acid. Br J Pharmacol. 1998;124:391-399.

72. Zwilling D, Huang S, Sathyasaikumar KV, et al. Kynurenine 3-monooxygenase inhibition in blood ameliorates neurodegeneration. Cell. 2012;145:863-874.
73. Okamoto S, Pouladi MA, Talantova $M$, et al. Balance between synaptic versus extrasynaptic NMDA receptor activity influences inclusions and neurotoxicity of mutant huntingtin. Nat Med. 2010;15:1407-1413.

74. Szalardy L, Zadori D, Toldi J, et al. Manipulating kynurenic acid levels in the brain - on the edge between neuroprotection and cognitive dysfunction. Curr Top Med Chem. 2012;12:1797-1806.

75. Ball HJ, Sanchez-Perez A, Weiser S, et al. Characterization of an indoleamine 2,3-dioxygenase-like protein found in humans and mice. Gene. 2007;396:203-213.

76. Metz R, DuHadaway JB, Kamasani U, et al. Novel tryptophan catabolic enzyme IDO2 is the preferred biochemical target of the antitumor indoleamine 2,3-dioxygenase inhibitory compound D-1-methyl-tryptophan. Cancer Res. 2007;67:7082-7087.

77. Yuasa HJ, Ball HJ. Indoleamine 2,3-dioxygenases with very low catalytic activity are well conserved across kingdoms: IDOs of basidiomycota. Fungal Genet Biol [Internet]. 2013;56:98-106.

78. Ball HJ, Yuasa HJ, Austin CJD, et al. Indoleamine 2,3-dioxygenase-2; a new enzyme in the kynurenine pathway. Int J Biochem Cell Biol. 2009;41:467-471.

79. Yuasa HJ, Ball HJ, Fern Y, et al. Characterization and evolution of vertebrate indoleamine 2,3-dioxygenases IDOs from monotremes and marsupials. Comp Biochem Physiol Part B. 2009;153:137-144.

80. van Baren N, Van den Eynde B. Tryptophan-degrading enzymes in tumoral immune resistance. Front Immunol. 2015;6:1-9.

81. Smith AK, Simon J, Gustafson $E$, et al. Association of a polymorphism in the indoleamine-2,3- dioxygenase gene and interferon-a-induced depression in patients with chronic hepatitis C. Mol Psychiatry [Internet]. 2012;17:781-789. Available from: http://www.nature.com/mp/journal/v17/n8/abs/mp201167a.html

82. Munn D, Zhou M, Attwood J, et al. Prevention of allogeneic fetal rejection by tryptophan catabolism. Science [Internet]. 1998;281:1191-1193. Available from: http://www.ncbi.nlm.nih.gov/ pubmed/9712583

83. Badawy AA-B, Namboodiri AMA, Moffett JR. The end of the road for the tryptophan depletion concept in pregnancy and infection. Clin Sci. 2016;130:1327-1333.

84. Yeung AWS, Terentis AC, King NJC, et al. Role of indoleamine 2,3-dioxygenase in health and disease. Clin Sci. 2015;129:601-672.

85. Baban B, Chandler $P$, Sharma $M$, et al. IDO activates regulatory $\mathrm{T}$ cells and blocks their conversion into TH17-like $\mathrm{T}$ cells. J Immunol. 2009;183:2475-2483.

86. Sharma MD, Huang L, Choi J-H, et al. An inherently bi-functional subset of FOxp3+ T helper cells is controlled by the transcription factor Eos. Immunitiy. 2013;38:998-1012.

87. Munn DH, Sharma MD, Baban B, et al. GCN2 kinase in T cells mediates proliferative arrest and anergy induction in response to indoleamine 2,3-dioxygenase. Immunity. 2005;22:633-642.

88. Terness P, Bauer TM, Röse L, et al. Inhibition of allogeneic T cell proliferation by indoleamine 2,3-dioxygenase - expressing dendritic cells: mediation of suppression by tryptophan metabolites. J Exp Med. 2002;196:447-457.

89. Scott GN, DuHadaway J, Pigott E, et al. The immunoregulatory enzyme IDO paradoxically drives B cell-mediated autoimmunity. J Immunol. 2010;182:7509-7517.

90. Liu H, Huang L, Bradley J, et al. GCN2-dependent metabolic stress is essential for endotoxemic cytokine induction and pathology. Mol Cell Biol. 2014;34:428-438.

91. Wang $X-F$, Wang $H-S$, Wang $H$, et al. The role of indoleamine 2,3-dioxygenase (IDO) in immune tolerance: focus on macrophage polarization of THP-1 cells. Cell Immunol. 2014;289:42-48.

92. El-Zaatari M, Chang Y, Zhang M, et al. Tryptophan catabolism restricts IFN- $\gamma$-expressing neutrophils and clostridium difficile immunopathology. J Immunol. 2014;193:807-816.

93. Boasso A, Herbeuval J-P, Hardy AW, et al. HIV inhibits CD4+ T-cell proliferation by inducing indoleamine 2,3-dioxygenase in plasmacytoid dendritic cells. Immunobilologyology. 2007;109:3351-3360. 
94. Gaelings L, Söderholm S, Bugai A, et al. Regulation of kynurenine biosynthesis during influenza virus infection. FEBS J. 2017;284:222-236.

95. Zhou L, Chen H, Wen Q, et al. Indoleamine 2,3-dioxygenase expression in human inflammatory bowel disease. Eur J Gastroenterol Hepatol. 2012;24:695-701.

96. Kwidzinski E, Aktas O, Richter D, et al. Indolamine 2,3-dioxygenase is expressed in the CNS and down-regulates autoimmune inflammation. FASEB J. 2005;19:1-19.

- The work of Kwidzinski et al. points out the dangers of IDO inhibition in a mouse model of multiple sclerosis.

97. Munn DH. Indoleamine 2,3-dioxygenase, tumor-induced tolerance and counter-regulation. Curr Opin Immunol. 2006;18:220-225.

1140 98. Widner B, Leblhuber F, Fuchs D. Increased neopterin production and tryptophan degradation in advanced Parkinson's disease. J Neural Transm. 2002;109:181-189.

99. Guillemin GJ, Brew BJ, Noonan CE, et al. Indoleamine 2,3 dioxygenase and quinolinic acid immunoreactivity in Alzheimer's disease hippocampus. Neuropathol Appl Neurobiol. 2005;31:395-404.

100. Campesan S, Green EW, Breda C, et al. The kynurenine pathway modulates neurodegeneration in a drosophila model of Huntington's disease. Curr Biol. 2011;21:961-966.

.. The findings of Campesan et al. show that genetic inhibiton of the Tdo2 enzyme (which can be considered an analogue of human IDO) in a Drosophila melanogaster HD model is protective against mutant $\mathrm{Htt}$ protein neurotoxicity.

101. Mazarei G, Budac DP, Lu G, et al. The absence of indoleamine 2,3-dioxygenase expression protects against NMDA receptor-mediated excitotoxicity in mouse brain. Exp Neurol J. 2013;249:144-148.

.. Mazarei and collegues showed that the lack of Ido gene in a mouse model resulted in decreased NMDA receptormediated toxicity.

1160 102. Guidetti P, Schwarcz R. 3-Hydroxykynurenine potentiates quinolinate but not NMDA toxicity in the rat striatum. Eur J Neuro. 1999;11:3857-3863.
103. Stone TW, Forrest CM, Stoy N, et al. Involvement of kynurenines in Huntington's disease and stroke-induced brain damage. J Neu. 2012;119:261-274.

104. Wurster CD, Ludolph AC. Antisense oligonucleotides in neurological disorders. Ther Adv Neurol Disord. 2018;11:1-19.

105. Tabrizi S, Leavitt B, Kordasiewicz $\mathrm{H}$, et al. Effects of IONIS-HTTRx in patients with early Huntington's disease, results of the first HTTlowering drug trial (CT.002). Neurology. 2018;90:(15 Supplement).

106. Maes M, Meltzer HY, Bosmans E, et al. Relationships between lower plasma L-tryptophan levels and immune-inflammatory variables in depression. Psychiatry Res. 1993;49:151-165.

- The work of Maes and collegues supports the notion that depressive symproms are amerliorated via IDO inhibition, as peripherial inflammation (an inducer of IDO expression) was shown to contribute to the appearance of depressive symptoms.

107. Wichers MC, Maes M. The role of indoleamine 2,3-dioxygenase (IDO) in the pathophysiology of interferon-a-induced depression. J Psychiatry Neurosci. 2004;29:11-17.

108. Yang G, Lu X, Yuan L. Biochimica et biophysica acta LncRNA: a link between RNA and cancer. BBA Gene Regul Mech [Internet]. 2014;1839:1097-1109.

109. Jasperson LK, Bucher C, Panoskaltsis-Mortari A, et al. Indoleamine 2,3-dioxygenase is a critical regulator of acute graft-versus-host disease lethality. Blood. 2008;111:3257-3266.

110. Reniers J, Meinguet C, Moineaux L, et al. Synthesis and inhibition study of monoamine oxidase, indoleamine 2,3-dioxygenase and tryptophan 2,3-dioxygenase by 3,8-substituted $5 \mathrm{H}$-indeno[1,2-c] pyridazin-5-one derivatives. Eur J Med Chem. 2011;46:6104-6111.

111. Jiang $T$, Sun $Y$, Yin $Z$, et al. Research progress of indoleamine 2,3-dioxygenase inhibitors. Future Med Chem. 2015;7:185-201.

- This review of developed IDO inhibitors, of which two have already reached clinical trials in cancer therapy, strenghtens the notion of IDO inhibition as a therapeutic approach.
1165

AQ4

1170

1175

1180

1185

1190 